\title{
Three-dimensional assessment of papillary muscle displacement in a porcine model of ischemic mitral regurgitation
}

\author{
Henrik Jensen, MD, PhD, ${ }^{\text {a,c }}$ Morten O. Jensen, $\mathrm{PhD},{ }^{\mathrm{a}, \mathrm{f}}$ Morten H. Smerup, $\mathrm{PhD},{ }^{\mathrm{a}}$ Steffen Ringgaard, $\mathrm{PhD},{ }^{\mathrm{b}}$ \\ Thomas S. Sørensen, PhD, ${ }^{c, d}$ Niels T. Andersen, PhD, ${ }^{\mathrm{e}}$ Per Wierup, MD, PhD, ${ }^{\mathrm{a}}$ J. Michael Hasenkam, MD, \\ $\mathrm{PhD}, \mathrm{DMSc},{ }^{\mathrm{a}}$ and Sten L. Nielsen, MD, PhD, DMSc ${ }^{\mathrm{a}}$
}

\begin{abstract}
Objective: Papillary muscle displacement relative to mitral annulus is pivotal in chronic functional ischemic mitral regurgitation. Analysis of 3-dimensional papillary muscle displacement has relied on invasive measurement. In this study, we used noninvasive clinically applicable 3-dimensional morphology cardiac magnetic resonance imaging to define papillary muscle position in a 3-dimensional matrix.
\end{abstract}

\begin{abstract}
Methods: Fifty pigs (approximately $50 \mathrm{~kg}$ ) were subjected to posterolateral myocardial infarction and tachycardiac stress. Fourteen animals survived 6 weeks: 10 acquired chronic functional ischemic mitral regurgitation at least grade II and 4 did not. Animals were examined by 3-dimensional morphology cardiac magnetic resonance imaging, and dedicated software enabled assessment of anterior and posterior papillary muscle positions relative to anterior and posterior trigones and posterior mitral annulus. Animals with functional ischemic mitral regurgitation were compared with those without and with 10 healthy controls.
\end{abstract}

Results: Relative to controls, animals with functional ischemic mitral regurgitation at end systole had significantly higher displacements of the posterior papillary muscle from anterior and posterior trigones in lateral and posterior directions, and of anterior papillary muscle from anterior and posterior trigones in apical direction. Relative to animals without functional ischemic mitral regurgitation, there was significantly higher posterior papillary muscle displacement from posterior trigone in lateral direction. Interpapillary muscle distance was the strongest predictor of regurgitant volume $\left(r^{2}=0.85, P<.001\right)$.

Conclusions: Three-dimensional morphology cardiac magnetic resonance imaging enabled detailed analysis of local left ventricular remodeling effects causing functional ischemic mitral regurgitation. (J Thorac Cardiovasc Surg 2010;140:1312-8)

Chronic functional ischemic mitral regurgitation (FIMR) is observed in $20 \%$ to $25 \%$ of patients after acute myocardial infarction, ${ }^{1}$ and long-term survival is directly related to its severity. ${ }^{2}$ FIMR at the time of coronary artery bypass grafting increases mortality as much as 4-fold even with revasculari-

From the Department of Cardiothoracic and Vascular Surgery $\mathrm{T},{ }^{\mathrm{a}}$ and The Magnetic Resonance Imaging Research Centre, ${ }^{\mathrm{b}}$ Aarhus University Hospital, Skejby, Denmark; the Institute of Clinical Medicine ${ }^{c}$ and the Departments of Computer Science, ${ }^{\mathrm{d}}$ and Biostatistics (Faculty of Health Sciences), ${ }^{\mathrm{e}}$ Aarhus University, Århus, Denmark; and the Department of Biomedical Engineering, ${ }^{\mathrm{f}}$ Engineering College of Aarhus, Århus, Denmark.

Supported by The Danish Heart Association (grants 06-4-B409-A895-2287 and 07-4B409-A1377-22369), the Lundbeck Foundation, the Korning Foundation, the Købmand Svend Hansen and Ina Hansens Foundation, the Aarhus University Research Initiative, the 17.12.1981 Foundation, the Lily Benthine Lund's Foundation, the Raimond og Dagmar Ringgaard-Bohn's Foundation, the Snedkermester Sofus Jacobsen og Hustru's Foundation, the Bohn's Foundation, the Direktør Jakob Madsen Foundation, the Alfred Helsted Foundation, the Augustinus Foundation, the Asta Florida Bolding Foundation, the Bønnelycke Foundation, the Eva og Robert Voss Hansens Foundation, and the Frode V. Nyegaard Foundation.

Disclosures: None.

Presented at the World Society of Cardio-Thoracic Surgery, Kos, Greece, May 2008. Received for publication Sept 8, 2009; revisions received Nov 9, 2009; accepted for publication Dec 31, 2009; available ahead of print March 29, 2010.

Address for reprints: Henrik Jensen, MD, PhD, Senior Registrar, Department of Cardiothoracic and Vascular Surgery T and Institute of Clinical Medicine, Aarhus University Hospital, Skejby, 8200, Denmark (E-mail: hjensen@dadlnet.dk).

$0022-5223 / \$ 36.00$

Copyright (c) 2010 by The American Association for Thoracic Surgery doi:10.1016/j.jtcvs.2009.12.042 zation. ${ }^{3}$ Furthermore, persistent or recurrent FIMR has been reported in $13 \%$ to $59 \%$ of patients late after standard downsized ring annuloplasty, ${ }^{4}$ and optimal surgical treatment is therefore still debatable. ${ }^{5}$ To improve surgical outcome the fundamental mechanisms of chronic FIMR have been examined. Although the exact mechanism causing chronic FIMR is still elusive, clinical $^{6}$ and experimental ${ }^{7}$ studies have focused on 2 central pathways, annular dilatation and papillary muscle (PM) displacement. Annular dilatation impedes leaflet coaptation by pulling the leaflets apart, most notably in septolateral directions (Carpentier type I dysfunction). The posterior PM has been shown to be displaced away from the annulus in the lateral and posterior directions in FIMR experimental models ${ }^{7}$ and in patients. ${ }^{8} \mathrm{PM}$ displacement causes mitral valve tethering into the left ventricle (LV), which impairs coaptation (Carpentier type IIIb dysfunction). Posterior PM displacement ${ }^{9}$ and continued LV remodeling ${ }^{10}$ have been shown to predict recurrence of FIMR after ring annuloplasty. Because standard downsized ring annuloplasty primarily addresses annular dilatation and not PM displacement, Kron and colleagues ${ }^{11}$ introduced surgical PM relocation as an adjunct procedure to downsized ring annuloplasty to relieve leaflet tethering and potentially improve durability of ring annuloplasty. PM geometry relative to mitral annular structures varies among patients, however, depending on 


\author{
Abbreviations and Acronyms \\ 3D $=3$-dimensional \\ FIMR = functional ischemic mitral valve \\ regurgitation \\ $\mathrm{LV} \quad=$ left ventricle \\ MRI = magnetic resonance imaging \\ $\mathrm{PM}=$ papillary muscle \\ $\mathrm{RegVol}=$ regurgitation volume
}

biologic variation and severity of remodeling. Therefore a detailed preoperative 3-dimensional (3D) analysis of the entire mitral valve apparatus is mandatory for planning surgical strategy. Experimentally, this has been achieved by epicardial ultrasonography, ${ }^{12}$ videofluoroscopy, ${ }^{13}$ and sonomicrometry. ${ }^{14}$ These techniques, however, require direct surgical access to the heart and therefore lack clinical applicability. In the clinical setting, 2-dimensional echocardiography is the preferred method of assessing PM position, and 3D echocardiography has recently been introduced ${ }^{15}$; however, assessment of discrete geometric changes in PM geometry requires very precise $3 \mathrm{D}$ positioning of scan planes. Handheld echocardiographic probe positioning to assess subvalvular geometry is limited by the need for an appropriate acoustic window and by operator experience, inducing high risks of intraobserver and interobserver variability. Cardiac magnetic resonance imaging (MRI) provides superior image quality and allows operator-independent positioning of scan planes with great accuracy in 3 dimensions without the need for an acoustic window. In this study, we used a noninvasive 3D morphology cardiac MRI technique ${ }^{16}$ for detailed geometric analysis of papillary muscle position in a pig model of FIMR. We aimed to identify the subvalvular geometric culprits responsible for the development of FIMR to provide a surgical planning tool for dedicated correction of FIMR.

\section{MATERIALS AND METHODS}

This study was conducted as a long-term intervention-control study on female Danish Landrace pigs. All animal experiments were conducted according to the guidelines given by the Danish Inspectorate for Animal Experimentation and after specific approval from this institution. Qualified animal caretaker personnel monitored the health status of the animals at all times during the study period. Analgesics were administered whenever an animal showed any sign of pain. In cases of refractory pain or poor thriving, animals were humanely killed.

\section{Surgical Technique}

The chronic FIMR animal model has recently been developed at our institution. ${ }^{17,18}$ Figure 1 displays survival and inclusion of animals during the study period. Fifty pigs weighing approximately $50 \mathrm{~kg}$ each were anesthetized with intramuscular injections of midazolam $(0.5 \mathrm{mg} / \mathrm{kg})$ and ketamine $(5 \mathrm{mg} / \mathrm{kg})$. Then intravenous access was obtained through an ear vein, and etomidate (Hypnomidate, $0.6 \mathrm{mg} / \mathrm{kg}$ ) was given to allow intubation. The animal was coupled to a ventilator, and sevoflurane inhalation was adjusted to a minimum alveolar concentration of $1 \%$ for continuous an- esthesia. A continuous fentanyl drip $(10 \mu \mathrm{g} /[\mathrm{kg} \cdot \mathrm{h}])$ was used for analgesia. Transthoracic echocardiography was performed to confirm mitral competence. Six animals were excluded for mitral regurgitation at baseline. In the remaining 44 animals, a $7 \mathrm{~F}$ sheath was placed in the right common carotid artery with the Seldinger technique. Coronary angiography was for performed, and a posterolateral LV wall infarction was induced by placing 2 to 3 metal coils in the circumflex artery proximal to the first obtuse marginal. A loading dose of amiodarone $(300 \mathrm{mg}$ ) and potassium chloride (10 mEq) was given during the 20 minutes before induction of infarction as prophylaxis against ventricular arrhythmias, and pancuronium bromide $(0.2 \mathrm{mg} / \mathrm{kg})$ was given as muscle relaxant. Sixteen animals died of ventricular fibrillation refractory to direct current conversion. Forty-five minutes after coil placement, a pacemaker with a right ventricular lead was implanted; starting on postoperative day 10, pacing intervals were conducted to promote $\mathrm{LV}$ remodeling (2 sequences of 7 days of pacing at 160 beats/min and 7 days off). Cefuroxime ( $1.5 \mathrm{~g}$ ) was administered both preoperatively and postoperatively to prevent infection. Postoperative analgesia was achieved with intramuscular injections of flunixin (INN flunixine, $0.4 \mathrm{mg} / \mathrm{kg}$ daily for 4 days) and acetaminophen (INN paracetamol , $1 \mathrm{~g}$ twice daily for 14 days). Buprenorphine $(0.3 \mathrm{mg} / \mathrm{mL})$ was administered intramuscularly to animals showing signs of pain. Amiodarone ( $200 \mathrm{mg}$ for 5 days) was given to reduce the risk of arrhythmia. During the period of intermittent pacing, animals were given furosemide ( $40 \mathrm{mg}$ twice daily) to avoid pulmonary congestion and potassium chloride ( $750 \mathrm{mg}$ twice daily) to prevent potassium depletion. Fourteen animals died or were humanely killed because of severe symptoms of heart failure during the next 6 weeks. Fourteen animals survived 6 weeks to be returned to the laboratory and were sedated, intubated, and coupled to a ventilator as described previously.

The presence of a mitral regurgitation jet was assessed by transthoracic echocardiography (Vivid 7; GE Vingmed Ultrasound, Horten, Norway). Severity of mitral regurgitation was graded as I through IV according to the American Heart Association and American College of Cardiology guidelines. ${ }^{19}$ Ten animals were considered to have FIMR of at least grade II (FIMR-positive group), whereas 4 were not (FIMR-negative group). The animals were then examined with cardiac MRI and subsequently allocated to further study. Ten weight-matched healthy female pigs $(53 \mathrm{~kg})$ served as controls. These animals underwent transthoracic echocardiography and cardiac MRI. No control animals had mitral regurgitation.

\section{Cardiac MRI}

MRI was conducted with a Philips Achieva 1.5-T MR Scanner (Philips Medical Systems BV, Best, The Netherlands), with electrocardiography used to synchronize data acquisition. Cine images were used to assess hemodynamics and mitral valve leaflet geometry and were acquired with the Balanced Steady-State-Free-Precession sequence with 30 heart phases, a slice thickness of $5 \mathrm{~mm}$, a pixel size $2.0 \times 2.0 \mathrm{~mm}^{2}$, a repetition time of $3.2 \mathrm{~ms}$, an echo time of $1.6 \mathrm{~ms}$, and a flip angle of $65^{\circ}$. Aortic flow was assessed with a 2-dimensional phase-contrast sequence with a slice thickness of $8 \mathrm{~mm}$, a pixel size $2.5 \times 2.5 \mathrm{~mm}^{2}$, a repetition time of $5.1 \mathrm{~ms}$, an echo time of $3.1 \mathrm{~ms}$, a flip angle of $15^{\circ}$, and a velocity encoding of $100 \mathrm{~cm} / \mathrm{s}$, which prevented aliasing in all scans. No animals had aortic regurgitation. The 3D morphology scans were acquired with the Balanced Steady-State-Free-Precession sequence. The field-of-view was $330 \times 330 \times 130 \mathrm{~mm}^{3}$, the repetition time $4.0 \mathrm{~ms}$, the echo time $2.0 \mathrm{~ms}$, and the flip angle $90^{\circ}$. Online identification of end diastole and end systole was necessary for planning of 3D morphology scans. End diastole was defined as the time of R-wave onset in the electrocardiogram, and end systole was defined as the time of maximum septal contraction.

\section{Hemodynamics}

LV stroke volume was obtained by subtracting LV volumes at end diastole and end systole on the basis of a 12-slice cine short-axis scan covering the LV from the apex to the center of the atrium. Mitral regurgitation volume (RegVol) and regurgitation fraction was obtained by subtracting the LV 


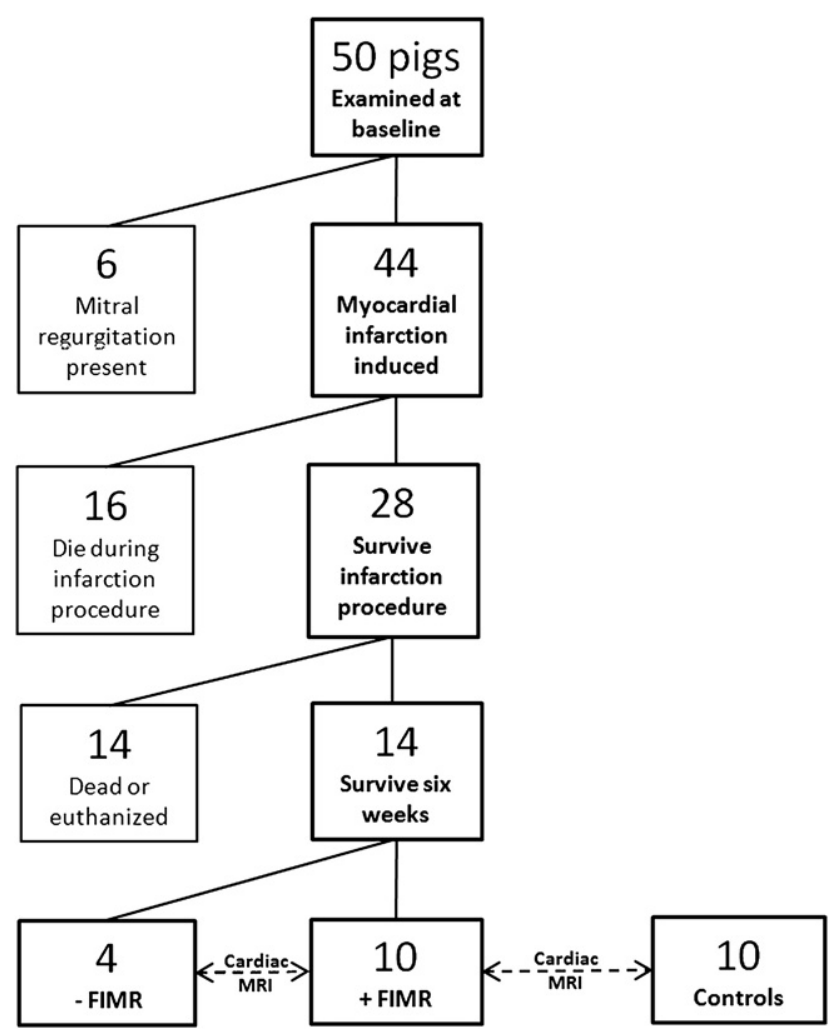

FIGURE 1. Survival and inclusion. MRI, Magnetic resonance imaging; FIMR, functional ischemic mitral regurgitation less than grade II; $+F I M R$, functional ischemic mitral regurgitation of at least grade II.

stroke volume from the aortic flow. The LV sphericity index was calculated as the ratio between the LV volume and a sphere with a diameter corresponding to the base to apex distance on a long-axis cine scan at end diastole and end systole.

\section{Papillary Muscle Position}

The 3D morphology cardiac MRI was performed with isotropic resolution of $1.72 \mathrm{~mm}$ along the 3 main axes (Figure 2, A). This allowed extraction of any imaging plane within the imaging volume (Cardiac3D; Systematic Software Engineering A/S, Århus, Denmark). ${ }^{16,18}$ The software used to analyze the 3D morphology scans enabled identification of any plane in the imaging volume, thereby allowing identification of the mitral annular plane, which was then translated into the left ventricle to define the papillary muscle tips as the first substantial part seen. The spatial positions of the anterior and posterior PMs, the anterior and posterior trigones, and the posterior mitral annulus were defined at end diastole and end systole, and corresponding Cartesian coordinates were transferred to mathematic analysis software (LabVIEW 8.0; National Instruments, Austin, Tex). All PM distances to trigone points (Figure 2, B), and annular, anteroposterior, and septolateral planes (Figure 2, $C$ ) were calculated.

\section{Statistics}

Statistical analysis was performed by a professional biostatistician (N.T.A.). Data from the control group and the FIMR-positive animals were tested for normal distribution. The low number of animals in the FIMR-negative group made it difficult to test the assumption of normal distribution; however, these animals represented a mix between control and FIMR-positive animals, and we therefore assumed that normal distribution was also present in the FIMR-negative group. Data are presented as

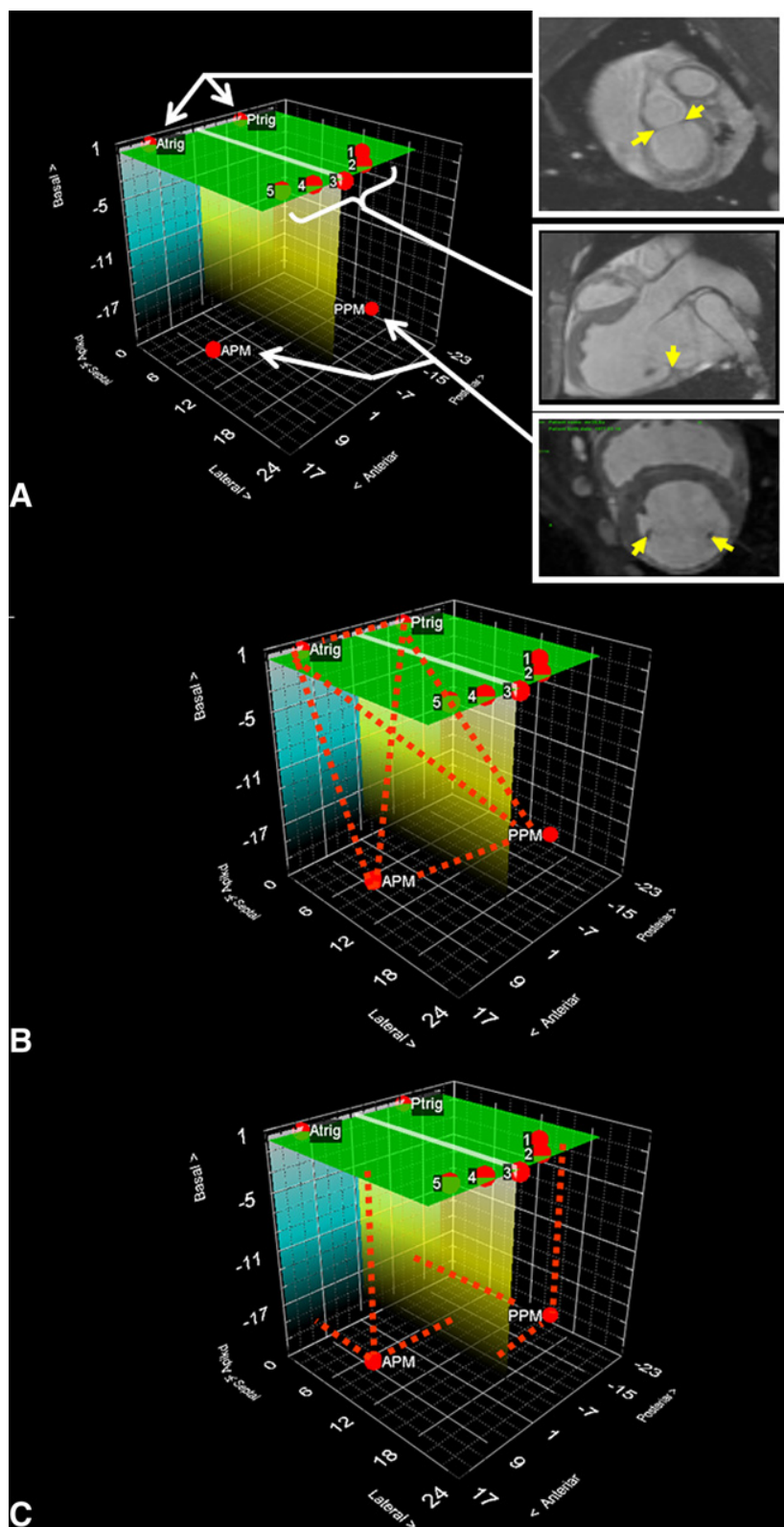

FIGURE 2. Three-dimensional morphology cardiac magnetic resonance imaging. Magnetic resonance imaging coordinates for trigone, annulus, and papillary muscle positions (yellow arrows) were transferred to dedicated mathematic analysis software. Annular plane (green) was defined by trigones and least squares fit to annular hinge points. Anteroposterior plane (blue) was defined perpendicular to annular plane and passing through both trigones. Septolateral plane (yellow) was defined perpendicular to annular and anteroposterior planes and passing through point midway between trigones. Ptrig, Posterior trigone; Atrig, anterior trigone; PPM, posterior papillary muscle; $A P M$, anterior papillary muscle.

mean $\pm \mathrm{SD}$. The control group and FIMR-positive animals were compared with the Student $t$ test. FIMR-positive and -negative animals were also compared with the Student $t$ test.

For the FIMR-positive animals at end systole, simple linear regression was used to determine which geometric parameter of papillary muscle position showed significant correlation with RegVol. Multiple linear 


\begin{tabular}{|c|c|c|c|c|c|}
\hline & \multirow[b]{2}{*}{ Control $(n=10)$} & \multirow[b]{2}{*}{ FIMR-positive $(\mathbf{n}=10)$} & \multirow[b]{2}{*}{ FIMR-negative $(n=4)$} & \multicolumn{2}{|c|}{$P$ value (vs FIMR-positive) } \\
\hline & & & & Control & FIMR-negative \\
\hline Weight $(\mathrm{kg})$ & $53.0 \pm 3.8$ & $54.6 \pm 2.8$ & $52.8 \pm 3.1$ & $>.20$ & $>.20$ \\
\hline LV ED volume (mL) & $106.7 \pm 17.1$ & $175.8 \pm 31.7$ & $141.6 \pm 18.6$ & $<.001$ & .069 \\
\hline LV ES volume (mL) & $50.5 \pm 16.4$ & $109.3 \pm 29.6$ & $90.0 \pm 20.9$ & $<.001$ & $>.20$ \\
\hline LV stroke volume (mL) & $56.3 \pm 8.6$ & $66.5 \pm 8.3$ & $51.4 \pm 11.0$ & .014 & .016 \\
\hline LV ejection fraction $(\%)$ & $53.4 \pm 9.2$ & $38.6 \pm 6.8$ & $36.8 \pm 8.4$ & $<.001$ & $>.20$ \\
\hline Heart rate (beats/min) & $82.5 \pm 18.9$ & $69.9 \pm 13.6$ & $81.3 \pm 16.2$ & .10 & .20 \\
\hline Cardiac output (L/min) & $4.6 \pm 1.0$ & $4.7 \pm 1.2$ & $4.1 \pm 0.9$ & $>.20$ & $>.20$ \\
\hline Aortic flow (mL/heart beat) & $52.1 \pm 11.3$ & $48.7 \pm 10.7$ & $46.7 \pm 12.2$ & $>.20$ & .77 \\
\hline Regurgitant volume (mL) & $4.2 \pm 9.3$ & $17.8 \pm 11.6$ & $4.7 \pm 2.4$ & $<.001$ & .049 \\
\hline Regurgitant fraction $(\%)$ & $7.1 \pm 15.1$ & $26.2 \pm 15.2$ & $9.7 \pm 4.9$ & 0.011 & .059 \\
\hline ED sphericity index & $40.6 \pm 5.8$ & $59.2 \pm 10.8$ & $57.3 \pm 11.9$ & $<.001$ & $>.20$ \\
\hline ES sphericity index & $26.7 \pm 6.5$ & $48.9 \pm 11.6$ & $49.9 \pm 12.7$ & $<.001$ & $>.20$ \\
\hline
\end{tabular}

All values mean \pm SD. FIMR, Functional ischemic mitral regurgitation; $L V$, left ventricular; $E D$, end-diastolic; $E S$, end-systolic.

regression was then used to determine which of these parameters was the strongest predictor of RegVol.

All authors had full access to the data and take responsibility for its integrity. All authors have read and agree to the article as written.

\section{RESULTS}

\section{Echocardiography}

The severity of mitral regurgitation in FIMR-positive animals was on average grade II.

\section{Cardiac MRI}

LV function. Relative to the control animals, the FIMRpositive animals had significantly higher LV end-diastolic volume, LV end-systolic volume, LV stroke volume, RegVol, regurgitation fraction, and end-diastolic and endsystolic sphericity indices. They also had lower LV ejection fraction. Compared with the FIMR-negative animals, the FIMR-positive group had significantly higher LV stroke volume and RegVol (Table 1).

PM position. Relative to the control animals, the FIMRpositive animals had significantly greater distances from each papillary muscle to each trigone and higher inter-PM distances at both end diastole and end systole. The posterior PM was significantly displaced in the lateral and posterior axis directions at both end diastole and end systole. The anterior PM was displaced significantly in the apical direction at end systole. Relative to the FIMR-negative animals, the FIMR-positive animals had significantly greater posterior PM displacement in the lateral direction and away from the posterior trigone at both end diastole and end systole. Furthermore, the intertrigone distance was significantly greater at end diastole (Table 2).

\section{Determinants of Mitral RegVol}

Simple linear regression revealed a significant positive correlation of RegVol with inter-PM distance $\left(r^{2}=0.84\right.$, $P<.001)$ and apical displacement of the anterior PM $\left(r^{2}=.56, P=.01 ;\right.$ Figure 3$)$. No other variables contributed significantly. Furthermore, these distances were significantly correlated with each other $\left(r^{2}=.58, P=.01\right)$. Inclusion of the 2 variables in a multiple linear regression model, however, revealed that RegVol was only significantly correlated with inter-PM distance $(P=.009)$ and not with apical displacement of the anterior PM $(P>.20)$.

\section{DISCUSSION}

Previous observations with this chronic FIMR model revealed Carpentier type I dysfunction as a result of annular dilatation and IIIb leaflet dysfunction as a result of leaflet tethering. ${ }^{18}$ To determine the key geometric culprits of anterior and posterior PM displacement causing leaflet tethering, we used a noninvasive 3D morphology cardiac MRI technique. Our analysis revealed that relative to a healthy control group, the FIMR-positive animals had the posterior PM displaced significantly away from the anterior PM and both trigones in posterior and lateral directions. Furthermore, the anterior PM was displaced significantly away from both trigones in the apical direction. The anatomic substrate was a localized posterolateral LV wall infarction that expanded with time as a result of tachycardiac stress. Accordingly, we demonstrated a positive correlation between inter-PM muscle distance and RegVol. Furthermore, significantly greater posterior PM displacement away from the posterior trigone in the lateral direction was observed in the FIMR-positive animals than in the FIMR-negative animals. These findings confirm the geometric culprits identified in the ovine chronic FIMR model ${ }^{7}$ and show that the clinically reported ${ }^{20}$ key role of posterior PM displacement in patients with FIMR is also valid in our FIMR model. The apical displacement of the anterior PM may be explained by global LV dilatation caused by remote remodeling effects after regional myocardial infarction, LV volume overload from FIMR, and the LV-dilating effect of 
TABLE 2. Papillary muscle position

\begin{tabular}{|c|c|c|c|c|c|}
\hline & \multirow[b]{2}{*}{ Control $(n=10)$} & \multirow[b]{2}{*}{ FIMR-positive $(\mathbf{n}=10)$} & \multirow[b]{2}{*}{ FIMR-negative $(n=4)$} & \multicolumn{2}{|c|}{$P$ value (vs FIMR-positive) } \\
\hline & & & & Control & FIMR-negative \\
\hline \multicolumn{6}{|l|}{ End diastole } \\
\hline \multicolumn{6}{|l|}{ Distances to reference planes } \\
\hline Annular plane-APM & $18.7 \pm 2.7$ & $22.3 \pm 5.3$ & $21.0 \pm 2.9$ & 0.073 & $>0.20$ \\
\hline Annular plane-PPM & $23.4 \pm 5.7$ & $20.2 \pm 7.0$ & $21.1 \pm 2.1$ & $>0.20$ & $>0.20$ \\
\hline Anteroposterior plane-APM & $15.2 \pm 5.7$ & $19.4 \pm 7.7$ & $17.7 \pm 10.4$ & 0.18 & $>0.20$ \\
\hline Anteroposterior plane-PPM & $24.5 \pm 5.2$ & $33.4 \pm 5.9$ & $24.3 \pm 8.3$ & 0.002 & 0.037 \\
\hline Septolateral plane-APM & $17.6 \pm 2.8$ & $19.5 \pm 5.9$ & $16.5 \pm 3.9$ & $>0.20$ & $>0.20$ \\
\hline Septolateral plane-PPM & $8.0 \pm 4.3$ & $15.5 \pm 8.0$ & $16.9 \pm 4.8$ & 0.018 & $>0.20$ \\
\hline \multicolumn{6}{|l|}{ Distances to anatomic landmarks } \\
\hline APM-PPM & $28.0 \pm 4.7$ & $39.4 \pm 5.8$ & $36.9 \pm 3.4$ & $<0.001$ & $>0.20$ \\
\hline APM-anterior trigone & $25.6 \pm 4.0$ & $31.8 \pm 6.8$ & $29.1 \pm 7.8$ & 0.024 & $>0.20$ \\
\hline APM-posterior trigone & $38.0 \pm 3.5$ & $43.8 \pm 4.8$ & $39.4 \pm 4.0$ & 0.006 & 0.13 \\
\hline PPM-anterior trigone & $40.0 \pm 3.4$ & $48.4 \pm 6.0$ & $42.7 \pm 5.2$ & 0.001 & 0.12 \\
\hline PPM-posterior trigone & $35.2 \pm 2.2$ & $40.8 \pm 4.2$ & $33.7 \pm 4.9$ & 0.001 & 0.017 \\
\hline Anterior-posterior trigone & $21.7 \pm 3.3$ & $23.2 \pm 2.1$ & $20.2 \pm 2.0$ & 0.26 & 0.03 \\
\hline \multicolumn{6}{|l|}{ End systole } \\
\hline \multicolumn{6}{|l|}{ Distances to reference planes } \\
\hline Annular plane-APM & $16.7 \pm 3.4$ & $22.1 \pm 5.1$ & $20.3 \pm 2.5$ & 0.011 & $>0.20$ \\
\hline Annular plane-PPM & $20.4 \pm 4.2$ & $20.4 \pm 7.0$ & $19.6 \pm 2.3$ & $>0.20$ & $>0.20$ \\
\hline Anteroposterior plane-APM & $15.3 \pm 5.5$ & $19.5 \pm 7.8$ & $17.2 \pm 13.2$ & 0.18 & $>0.20$ \\
\hline Anteroposterior plane-PPM & $24.5 \pm 4.6$ & $33.4 \pm 4.5$ & $24.5 \pm 9.0$ & $<0.001$ & 0.026 \\
\hline Septolateral plane-APM & $14.4 \pm 3.0$ & $19.0 \pm 6.5$ & $15.5 \pm 5.5$ & 0.057 & $>0.20$ \\
\hline Septolateral plane-PPM & $7.1 \pm 3.0$ & $14.5 \pm 8.0$ & $15.6 \pm 9.1$ & 0.018 & $>0.20$ \\
\hline \multicolumn{6}{|l|}{ Distances to anatomic landmarks } \\
\hline APM-PPM & $22.6 \pm 3.9$ & $38.0 \pm 7.4$ & $33.8 \pm 3.3$ & $<0.001$ & $>0.20$ \\
\hline APM-anterior trigone & $23.7 \pm 4.8$ & $31.9 \pm 6.5$ & $30.1 \pm 11.7$ & 0.005 & $>0.20$ \\
\hline APM-posterior trigone & $33.8 \pm 4.8$ & $43.1 \pm 5.7$ & $37.7 \pm 3.8$ & 0.001 & $>0.20$ \\
\hline $\mathrm{PPM}$-anterior trigone & $36.4 \pm 3.4$ & $47.6 \pm 6.5$ & $42.4 \pm 4.5$ & $<0.001$ & 0.18 \\
\hline PPM-posterior trigone & $33.1 \pm 2.8$ & $40.7 \pm 4.4$ & $33.0 \pm 6.0$ & $<0.001$ & 0.02 \\
\hline Anterior-posterior trigone & $20.1 \pm 3.9$ & $22.1 \pm 2.4$ & $21.0 \pm 3.5$ & 0.20 & $>0.20$ \\
\hline
\end{tabular}

All values are mean $\pm \mathrm{SD}$ in millimeters. FIMR, Functional ischemic mitral regurgitation; $A P M$, anterior papillary muscle; PPM, Posterior papillary muscle.

tachycardiac stress. Even though it was not specifically investigated in this study, the asymmetric displacement of the PMs theoretically would especially distort the posteromedial aspects of the mitral valve corresponding to the P2-P3 segment of the posterior leaflet, in accordance with experimental and clinical observations. Furthermore, clinical studies have shown that it takes less global remodeling to produce chronic FIMR by asymmetric leaflet tethering than by symmetric leaflet tethering. ${ }^{6}$ This underlines the importance of addressing the 3D geometry of both PMs and suggests an important role in FIMR for anterior PM displacement as well as posterior PM displacement, which so far has been the center of attention. It is plausible that lateralization of leaflet tethering forces by posterolateral posterior PM displacement and apical anterior PM displacement in the FIMR-positive animals (increased inter-PM distance) diverted the central parts of the leaflets away from closure and thereby exacerbated the degree of incomplete mitral leaflet coaptation and FIMR. According to this theory, the less posterior PM displacement from the posterior trigone in lateral direction (and tendency of shorter inter-PM distance) in the FIMR-negative animals was not sufficient to distort the central part of the leaflets to produce a significant regurgitant jet.

Recent studies have shown that continued LV remodeling is associated with recurrence of FIMR after ring annuloplasty. ${ }^{10}$ Furthermore, it has been reported that ring annuloplasty in itself can augment leaflet tethering. ${ }^{21}$ In this light, it has been advocated that surgical repair that remodels subvalvular geometry should be added as an adjunct procedure to downsized ring annuloplasty in FIMR. A number of techniques have been proposed, including direct relocation of the posterior PM with a stitch from the PM tip to the annulus, ${ }^{11}$ folding the $\mathrm{LV}$ wall at the root of the posterior $\mathrm{PM},{ }^{22} \mathrm{PM}$ approximation, ${ }^{23}$ and epicardial balloon patch, ${ }^{24}$ among others. To optimize adjunct procedures to downsized ring annuloplasty, extensive preoperative mitral valve imaging to identify the culprit lesion in both the anterior and posterior PM geometry is mandatory. The noninvasive cardiac MRI technique used in this study provides a tool for such 


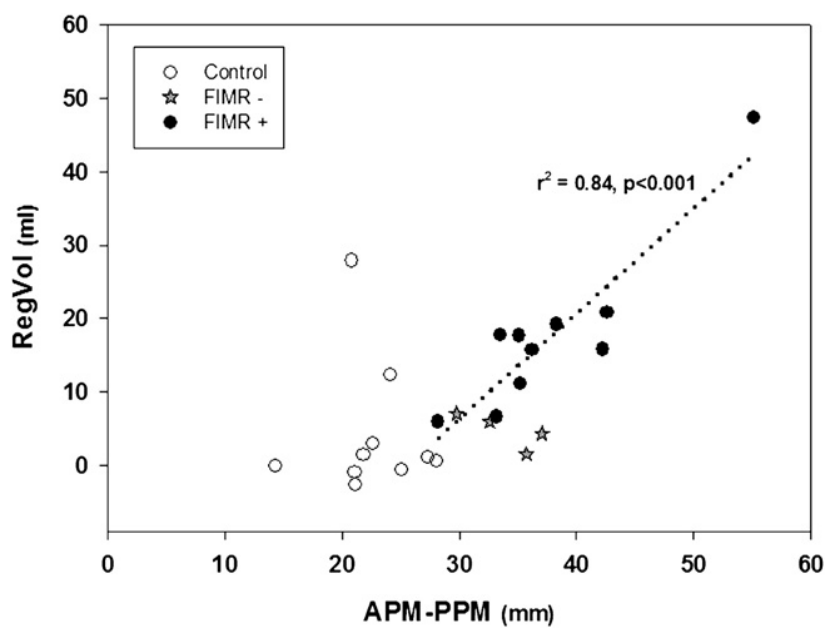

FIGURE 3. Correlation between anterior to posterior papillary muscle $(A P M-P P M)$ distance and regurgitant volume (RegVol). Of all end-systolic papillary muscle position parameters, anterior to posterior papillary muscle distance showed strongest correlation with regurgitant volume. -FIMR, Functional ischemic mitral regurgitation less than grade II; $+F I M R$, functional ischemic mitral regurgitation of at least grade II.

a comprehensive preoperative and postoperative assessment of 3D PM position. The results suggest that a subvalvular remodeling procedure targeting specific geometric culprits of PM displacement in this FIMR model should comprise an approximately 7- to $12-\mathrm{mm}$ relocation of the anterior and posterior PMs toward the trigones. Furthermore, mitral valve imaging should be repeated postoperatively to assess whether the planned subvalvular remodeling has actually been obtained. By comparing the outcome of surgery (freedom from recurrence of FIMR) with the actual obtained subvalvular remodeling, key elements in an optimal PM relocation procedure could potentially be identified.

\section{Limitations}

In this study, PM distances to defined anatomic landmarks in the mitral annulus were assessed from a Cartesian coordinate system with an external reference point (the MRI scanner). In parts of the analysis, however, 3 internal reference planes were defined according to anatomic and moving landmarks of the mitral valve apparatus (least squares fit to the mitral annulus). As pointed out by Dagum and colleagues, ${ }^{13}$ the use of internal reference planes can be problematic because differences in mitral annular anatomy among animals will result in different coordinate systems. Furthermore, a coordinate system that is based on annular points changes through the heart cycle because of the physiologic changes in mitral annular shape. In this study, we did not assess changes during the heart cycle; however, we did compare different animals with internal reference planes.

We examined FIMR in pig hearts with large transmural infarctions in LVs without collaterals but with tachycardia- induced dilatation. This FIMR model thus may represent a hybrid between true ischemic FIMR and tachycardiainduced cardiomyopathy and therefore may not fully reflect a clinical scenario with long-standing coronary artery atherosclerosis, collaterals, and coronary revascularization. Animals were sedated during examination, which may underestimate the severity of FIMR.

Assessment of mitral regurgitation with cardiac MRI has not yet been perfected. In this study, we quantified mitral regurgitation by subtracting the aortic flow from the stroke volume. Although this method is considered the criterion standard, ${ }^{25}$ it both is highly observer dependent and introduces a potential source of error, as evidenced in this study by the 2 animals in the control group (Figure 3 ) had high RegVol assessed by cardiac MRI but no regurgitant jet on Doppler echocardiography.

There were only 10 animals in each group, which limits the power of regression analysis with many explanatory variables. Furthermore, in the regression analysis of the anterior to posterior PM distance and RegVol, 1 FIMR-positive animal was an outlier (Figure 3), which contributed a great deal to making the correlation statistically significant.

This study contains a high number of statistical tests that induce a multiplicity problem. We believe, however, that the Bonferroni correction is conservative for hypothesisgenerating purposes. To accommodate the multiplicity problem, the $P$ values for each test are stated in tables and text to display the level of significance.

\section{CONCLUSIONS}

With data obtained by a noninvasive 3D morphology cardiac MRI technique, this comprehensive geometric analysis of the mitral valve complex identified increased inter-PM distance related to asymmetric displacement of the anterior and posterior PMs as an important and independent determinant of FIMR. When considering adjunct procedures to ring annuloplasty, such as PM relocation, this clinically applicable analytic technique potentially allows a more detailed exploration of the pathogenesis of FIMR and may prove a valuable clinical tool for preoperative planning of a surgical strategy that is based on quantitative data and not just intuition.

We thank the staff at the Institute for Clinical Medicine, Aarhus University, Department of Health Sciences, for excellent surgical facilities, the Påskehøjgaard Centre for taking excellent care of our animals, and the department of Cardiology at Aarhus University Hospital, Skejby, for kindly supplying us with explanted pacemakers. Furthermore, we thank St Jude Medical, Medtronic, and COOK Medical for supplying utilities for the experiments.

\section{References}

1. Bursi F, Enriquez-Sarano M, Jacobsen SJ, Roger VL. Mitral regurgitation after myocardial infarction: a review. Am J Med. 2006;119:103-12. 
2. Grigioni F, Enriquez-Sarano M, Zehr KJ, Bailey KR, Tajik AJ. Ischemic mitral regurgitation: long-term outcome and prognostic implications with quantitative Doppler assessment. Circulation. 2001;103:1759-64.

3. Adler DS, Goldman L, O'Neil A, Cook EF, Mudge GH Jr, Shemin RJ, et al. Longterm survival of more than 2,000 patients after coronary artery bypass grafting. Am J Cardiol. 1986;58:195-202.

4. Magne J, Senechal M, Dumesnil JG, Pibarot P. Ischemic mitral regurgitation: a complex multifaceted disease. Cardiology. 2009;112:244-59.

5. Adams DH, Filsoufi F, Aklog L. Surgical treatment of the ischemic mitral valve. J Heart Valve Dis. 2002;11(Suppl. 1):S21-5.

6. Agricola E, Oppizzi M, Maisano F, De Bonis M, Schinkel AF, Torracca L, et al. Echocardiographic classification of chronic ischemic mitral regurgitation caused by restricted motion according to tethering pattern. Eur J Echocardiogr. 2004;5: 326-34.

7. Tibayan FA, Rodriguez F, Zasio MK, Bailey L, Liang D, Daughters GT, et al. Geometric distortions of the mitral valvular-ventricular complex in chronic ischemic mitral regurgitation. Circulation. 2003;108. Suppl. 1:II116-21.

8. Yiu SF, Enriquez-Sarano M, Tribouilloy C, Seward JB, Tajik AJ. Determinants of the degree of functional mitral regurgitation in patients with systolic left ventricular dysfunction: a quantitative clinical study. Circulation. 2000;102:1400-6.

9. Kuwahara E, Otsuji Y, Iguro Y, Ueno T, Zhu F, Mizukami N, et al. Mechanism of recurrent/persistent ischemic/functional mitral regurgitation in the chronic phase after surgical annuloplasty: importance of augmented posterior leaflet tethering. Circulation. 2006;114(1 Suppl):I529-34.

10. Hung J, Papakostas L, Tahta SA, Hardy BG, Bollen BA, Duran CM, et al. Mechanism of recurrent ischemic mitral regurgitation after annuloplasty: continued LV remodeling as a moving target. Circulation. 2004;110(11 Suppl. 1):II85-90.

11. Kron IL, Green GR, Cope JT. Surgical relocation of the posterior papillary muscle in chronic ischemic mitral regurgitation. Ann Thorac Surg. 2002;74:600-1.

12. Liel-Cohen N, Guerrero JL, Otsuji Y, Handschumacher MD, Rudski LG, Hunziker PR, et al. Design of a new surgical approach for ventricular remodeling to relieve ischemic mitral regurgitation: insights from 3-dimensional echocardiography. Circulation. 2000;101:2756-63.

13. Dagum P, Timek TA, Green GR, Lai D, Daughters GT, Liang DH, et al. Coordinate-free analysis of mitral valve dynamics in normal and ischemic hearts. $\mathrm{Circu}$ lation. 2000;102(19 Suppl. 3):III62-9.

14. Gorman JH 3rd, Jackson BM, Gorman RC, Kelley ST, Gikakis N, Edmunds LH Jr. Papillary muscle discoordination rather than increased annular area facilitates mitral regurgitation after acute posterior myocardial infarction. Circulation. 1997;96(9 Suppl):II124-7.
15. Ryan LP, Jackson BM, Eperjesi TJ, Plappert TJ, St John-Sutton M, Gorman RC, et al. A methodology for assessing human mitral leaflet curvature using real-time 3-dimensional echocardiography. J Thorac Cardiovasc Surg. 2008;136:726-34.

16. Sorensen TS, Korperich H, Greil GF, Eichhorn J, Barth P, Meyer H, et al. Operator-independent isotropic three-dimensional magnetic resonance imaging for morphology in congenital heart disease: a validation study. Circulation. 2004; 110:163-9.

17. Bjerre M, Jensen H, Andersen JD, Ringgaard S, Smerup M, Wierup P, et al. Chronic ischemic mitral regurgitation induced in pigs by catheter-based coronary artery occlusion. J Heart Valve Dis. 2008;17:283-9.

18. Jensen H, Jensen M $\varnothing$, Ringgaard S, Smerup MH, Sorensen TS, Kim WY, et al. Geometric determinants of chronic functional ischemic mitral regurgitation: insights from three-dimensional cardiac magnetic resonance imaging. J Heart Valve Dis. 2008;17:16-23.

19. Zoghbi WA, Enriquez-Sarano M, Foster E, Grayburn PA, Kraft CD, Levine RA, et al. Recommendations for evaluation of the severity of native valvular regurgitation with two-dimensional and Doppler echocardiography. J Am Soc Echocardiogr. 2003; 16:777-802.

20. Kumanohoso T, Otsuji Y, Yoshifuku S, Matsukida K, Koriyama C, Kisanuki A, et al. Mechanism of higher incidence of ischemic mitral regurgitation in patients with inferior myocardial infarction: quantitative analysis of left ventricular and mitral valve geometry in 103 patients with prior myocardial infarction. $J$ Thorac Cardiovasc Surg. 2003;125:135-43.

21. Zhu F, Otsuji Y, Yotsumoto G, Yuasa T, Ueno T, Yu B, et al. Mechanism of persistent ischemic mitral regurgitation after annuloplasty: importance of augmented posterior mitral leaflet tethering. Circulation. 2005;112(9 Suppl):I396-401.

22. Terai H, Tao K, Sakata R. Surgical treatment for ischemic mitral regurgitation: strategy for a tethered valve. Ann Thorac Cardiovasc Surg. 2005;11:288-92.

23. Matsui Y, Suto Y, Shimura S, Fukada Y, Naito Y, Yasuda K, et al. Impact of papillary muscles approximation on the adequacy of mitral coaptation in functional mitral regurgitation due to dilated cardiomyopathy. Ann Thorac Cardiovasc Surg. 2005; 11:164-71.

24. Hung J, Guerrero JL, Handschumacher MD, Supple G, Sullivan S, Levine RA. Reverse ventricular remodeling reduces ischemic mitral regurgitation: echoguided device application in the beating heart. Circulation. 2002;106: 2594-600.

25. Lyne JC, Pennell DJ. Cardiovascular magnetic resonance in the quantitative assessment of left ventricular mass, volumes and contractile function. Coron Artery Dis. 2005;16:337-43. 